\title{
Parallels between embryo and cancer cell metabolism
}

\author{
Danielle G. Smith* and Roger G. Sturmey† ${ }^{1}$ \\ "Children's Cancer Research Group, Leeds Institute of Molecular Medicine, St. James's University Hospital, Beckett Street, Leeds LS9 7TF, U.K., and †Hull York \\ Medical School, Centre for Cardiovascular and Metabolic Research, University of Hull, Hull HU6 7RX, U.K.
}

\begin{abstract}
A key characteristic of cancer cells is the ability to switch from a predominantly oxidative metabolism to glycolysis and the production of lactate even when oxygen is plentiful. This metabolic switch, known as the Warburg effect, was first described in the 1920s, and has fascinated and puzzled researchers ever since. However, a dramatic increase in glycolysis in the presence of oxygen is one of the hallmarks of the development of the early mammalian embryo; a metabolic switch with many parallels to the Warburg effect of cancers. The present review provides a brief overview of this and other similarities between the metabolism in tumours and early embryos and proposes whether knowledge of early embryo metabolism can help us to understand metabolic regulation in cancer cells.
\end{abstract}

\section{Introduction}

The origins of the study of cancer cell metabolism can be traced back to the work of Warburg in the 1920s, who first showed that many tumours increase the consumption of glucose and production of lactate in the presence of oxygen (summarized by Warburg [1]). Although the cells of most tissues can switch from mitochondrial oxidative phosphorylation to glycolytic production of ATP under hypoxic conditions, cancer cells appear to adopt this metabolic phenotype as part of their normal function and in the presence of oxygen. This 'aerobic glycolysis' has been observed in tumours in vivo, and is used as a diagnostic tool [2]. However, cancer cells are not the only cells to be characterized by high aerobic glycolysis; such an effect has been described as the metabolic phenotype of rapidly proliferating cells generally [3].

Interestingly, aerobic glycolysis occurs in the early preimplantation mammalian embryo, an observation first hinted at by the work of Fridhandler [4]. Moreover, it is now apparent that this is not the only metabolic feature that is common to cancers and early embryos. In the present review, we provide an overview of key metabolic strategies of the early embryo as it undergoes preimplantation development, with emphasis on glucose, amino acids and fatty acids, and highlight some of the parallels with the superficial metabolic hallmarks of cancer progression.

Key words: blastocyst, embryo, glycolysis, pyruvate kinase M2, tumour

Abbreviations used: IVF, in vitro fertilization; MAGL, monoacylglycerol lipase; NEFA, nonesterified fatty acid; PEP, phosphoenolpyruvate; PGAM, phosphoglycerate mutase; PK, pyruvate kinase; PPP, pentose phosphate pathway; TCA, tricarboxylic acid.

${ }^{1}$ To whom correspondence should be addressed (email roger.sturmey@hyms.ac.uk).

\section{Early embryo development and metabolism}

During mammalian preimplantation development, the fertilized egg, or zygote, undergoes a series of mitotic divisions until it ultimately forms a blastocyst, a key developmental end point after which implantation occurs (Figure 1). Formation of the blastocyst is a dynamic period of development, characterized by an increase in ATP demand and met primarily through oxidative metabolism [5-7], which is relatively low during cleavage development, before rising 2-4-fold during the formation of the blastocyst.

The study of substrate utilization to satisfy ATP demand by early embryos has traditionally been driven by the desire to define the 'requirements' for embryos to be grown in vitro, with a view to improving success rates in clinical IVF (in vitro fertilization) [8]. A complementary aim to this has been attempts to discover non-invasive biomarkers of embryo viability $[9,10]$ and studies in this area have provided a good understanding of how the early embryo uses a number of key metabolic substrates, such as glucose, pyruvate, lactate, amino acids and fatty acids.

\section{Glucose metabolism in cancers and early embryos}

\section{Cancers}

Aerobic glycoloysis in tumours occurs despite vascularization or ready access to oxygen as is the case for haematological cancers. The biological explanation and mechanism underlying aerobic glycolysis are unclear. Although a number of cancers exhibit genetic defects in the mitochondrial proteins in the oxidative phosphorylation chain, the majority of cancers do not, and the origins of aerobic glycolysis are likely 
Figure 1 | A brief overview of preimplantation development from the one-cell zygote to the formation of a blastocyst

The fertilized zygote completes a number of mitotic divisions in a process called cleavage. With each progressive cleavage, the embryo replicates its nuclear component and increases cell number, but distributes cytoplasm among the daughter blastomeres, thus reducing the size of each individual cell with the net result of no overall change in total size, and biosynthesis largely limited to the production of membranes. After eight to ten rounds of cleavage, the blastomeres are no longer visible as individual cells as the cells compact on each other, coincident with the formation of cell-cell junctions; at this stage, the early embryo is referred to as a morula, which subsequently forms a blastocyst. This key stage in early development comprises the first cellular differentiation, resulting in the TE (trophectoderm) lineage and the ICM (inner cell mass). It is from the ICM that embryonic stem cells can be derived in vitro, whereas in situ these cells give rise to the embryo proper. Additionally, the blastocyst marks the start of true embryonic growth, protein synthesis, mitochondrial maturation and biogenesis and ion pumping to form the fluid-filled blastocoel cavity.

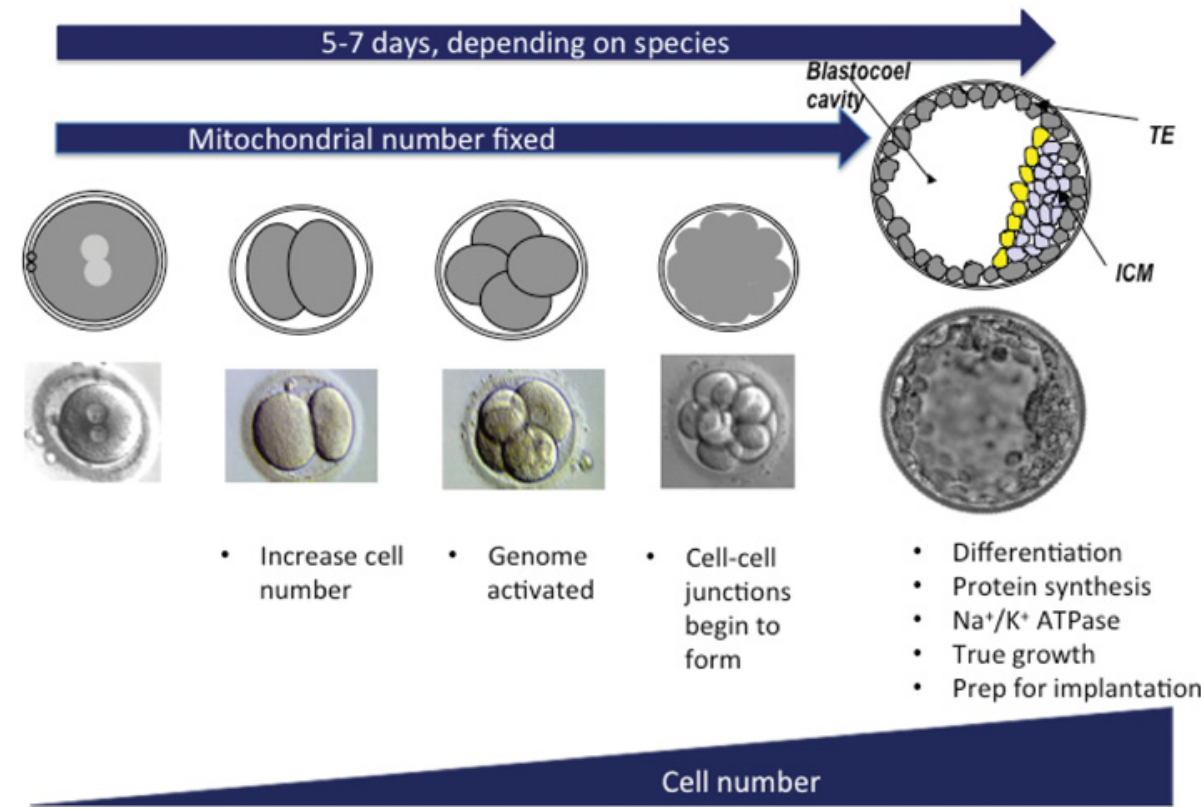

to be multifactorial $[11,12]$. By-products of glycolysis include large amounts of lactate and free acid $\left(\mathrm{H}^{+}\right)$, both of which are toxic to normal cells. However, cancer cells appear to select for this ability and thrive rather than undergo apoptosis. Thus the production of lactate leads to acute and chronic local acidosis, which may provide tumour cells with a selective advantage over neighbouring cells, which lack mechanisms to cope with a reduction in $\mathrm{pH}$ [2]. Acidification of the local environment may lead to the breakdown of extracellular matrix, disruption of gap junctions and ultimately promote invasion and metastasis.

\section{Embryos}

Similar to the aerobic glycolysis observed in a tumour cell, the metabolism of a blastocyst is typified by an increase in the consumption of glucose. This has been studied most extensively in the early embryos of mouse $[6,12,13]$, although it has also been reported in cow [7], pig [5,14] and human [15]. Coinciding with the rise in glucose consumption, mammalian blastocysts release significant quantities of lactate $[6,7,14]$. Glycolysis occurs at a high level (80-90\%) in embryos produced in vitro, but to a lesser extent in those produced in vivo and flushed from the female tract (44\% in mouse). This value of glycolysis increases within $1 \mathrm{~h}$ to close to the in vitro level [16], and, surprisingly, is increased in a high oxygen environment. Taken together, this may be interpreted that the glycolytic activity of blastocysts is independent of oxygen levels. In this regard, the appearance of an aerobic glycolytic phenotype as the mammalian embryo forms a blastocyst appears to be very similar to that of tumour cells. This notion was recently the subject of an authoritative review by Krisher and Prather [17]. The mammalian blastocyst must obviously implant in the uterus for successful pregnancy, and it is tempting to speculate that extracellular acidification mediated by glycolytic production of lactate plays a role in supporting this tissue implantation by the early embryo in a manner that mirrors tumour invasion as hypothesized by Gatenby and Gillies [2].

The idea that glycolysis increases in cancers and embryos solely to provide ATP may be misguided since glycolytic conversion of glucose into lactate is an inefficient way of producing ATP compared with the complete oxidation through the TCA (tricarboxylic acid) cycle. However, a compelling explanation for the increased need for 
glycolysis comes from Vander Heiden et al. [3], who argue that partitioning glucose through glycolysis may result in an increased production of glycolytic intermediaries, particularly glucose 6-phosphate, which can be shunted into the PPP (pentose phosphate pathway). In both tumour cells and early embryos, it is unlikely that the supply of substrates for ATP production will be limiting; it is much more probable that demand for carbon for biosynthesis may outweigh supply. Increased flux through the PPP may mediate this shortfall of carbon for biosynthesis.

Cells have differential requirements for nucleic acid and lipid precursors to support rapid proliferation [3] and the PPP is an essential source of ribose sugars required for nucleic acid synthesis, as well as $\mathrm{NAD}(\mathrm{P}) \mathrm{H}$, the reducing agent and cofactor in lipid biosynthesis. Furthermore, $\mathrm{NAD}(\mathrm{P}) \mathrm{H}$ is necessary for maintenance of cellular redox status by driving glutathione reductase activity [18], which reduces GSSG back to GSH, as well as the biosynthesis of GSH itself. GSH is ubiquitously expressed in tissues and is the most abundant non-enzymatic antioxidant in mammalian cells, including early embryos [19].

As described above, the developing blastocyst begins rapid proliferation, increases protein synthesis and true growth begins, and it is therefore likely that demand of important biosynthetic precursors is met in part by PPP activity. In the early embryo, Javed and Wright [20] reported that the proportion of glucose flowing through the PPP increases from approximately $7 \%$ during the cleavage stage to approximately $20 \%$ in the blastocyst, indicating the importance of this pathway in normal preimplantation development.

\section{Amino acids}

\section{Cancer cells}

Increased glutaminolysis has been described as a metabolic hallmark of cancer progression [21], and a simultaneous increase in glycolysis and glutaminolysis occurs in a number of rapidly proliferating cell types including cancer cells [22]. Glutamine is converted into glutamate by mitochondrial and cytoplasmic glutaminase, then converted into 2-oxoglutarate ( $\alpha$-ketoglutarate) catalysed by glumate dehydrogenase. 2 Oxoglutarate feeds directly into the TCA cycle, thus is an important cellular source of ATP, with as much as $50 \%$ of cellular ATP produced by this mechanism in some cancers [23]. However, similar to aerobic glycolysis, it is likely that increased glutaminolysis in tumours and other rapidly proliferating cells may have roles in addition to ATP supply. A number of tumour cells overexpress cytoplasmic malic enzyme [24] that converts malate into pyruvate, which can be exported from mitochondria, leading to a downstream production of NADPH required for other biosynthetic pathways and redox regulation [23]. Glutamine is a key source of nitrogen and carbon for biosynthetic processes and also plays an anaplerotic role in tumours, by generating citrate to replenish carbon atoms lost from the TCA cycle to lipid biosynthesis [25].

\section{Early embryos}

Provision of glutamine during embryo development in vitro leads to a dramatic improvement in development [26-29]. It is consistently depleted from the culture medium of early embryos in all species studied [10,30,31]. Interestingly, the pattern of consumption and release of a number of amino acids, including glutamine is predictive of embryo viability and may well form the basis of a biomarker for the selection of embryos for transfer in a clinical IVF setting. The metabolic roles of glutamine in early embryonic development include provision of ATP via conversion to 2-oxoglutarate and oxidative metabolism [32], involvement in biosynthetic processes [33] and an osmolyte.

The importance of glutaminolysis in early embryos and cancer cells is one of a number of similarities in usage of amino acids between these apparent disparate tissue types. With regard to other amino acids, in a recent technical tour de force, Jain et al. [34] performed a comprehensive analysis of the consumption and release of over 200 small-molecule metabolites by a range of cancer cell lines in vitro. The standout observation in this work was a switch from glycine release to glycine consumption that correlated strongly with cancer progression. An analogous pattern has been reported in early human embryos; those embryos that are unable to give a pregnancy release glycine into the culture medium. By contrast, viable embryos switch to a modest retention of glycine. An increased requirement for glycine during development of viable embryos and cancer progression may stem from a need for GSH to protect against oxidative damage. Although not the rate-limiting step, glycine is an important precursor for the synthesis of GSH; the final step of GSH biosynthesis is the addition of glycine to glutamyl cysteine catalysed by GSH synthetase.

\section{Fatty acid metabolism}

\section{Cancer cells}

The role of fatty acid metabolism in cancer cells has received very little attention, with the bulk of this limited research focusing on de novo synthesis of fatty acids [35] in tumorigenesis to provide the building blocks for membrane synthesis, signal transduction molecules and pro-tumorigenic lipid signalling molecules. Increased fatty acid synthesis in tumours is mediated in part by elevated levels of fatty acid synthase [36]. It has been reported that some highgrade, but not low-grade, tumours from multiple tissues express elevated levels of MAGL (monoacylglycerol lipase) which metabolizes glycerolipid monoacylglycerols from neutralized lipid stores. This process liberates NEFAs (nonesterified fatty acids) that can be converted into protumorigenic lipid transmitters which stimulate cell migration and tumorigenic activity [36]. Furthermore, inhibition of MAGL activity in aggressive tumour lines impaired migration and 
Figure 2 | Proposed scheme of the interaction of the Warburg effect enhanced glycolysis and regulation of gene expression Enhanced glycolysis and regulation of gene expression may exist in both early embryos and the cells of many cancers. Glucose metabolism through glycolysis is slowed by the expression of PKM2, which leads to a build-up of glycolytic precursors, particularly glucose 6-phosphate, which contributes to PPP for biosynthesis. ATP is generated from other sources such as fatty acids and amino acids. An additional role for PKM2 is via its activity as a transcription factor, where it regulates the expression of a number of developmentally important genes, which are also implicated in the progression of cancer. CK, creatine kinase; ETC, electron transport chain.

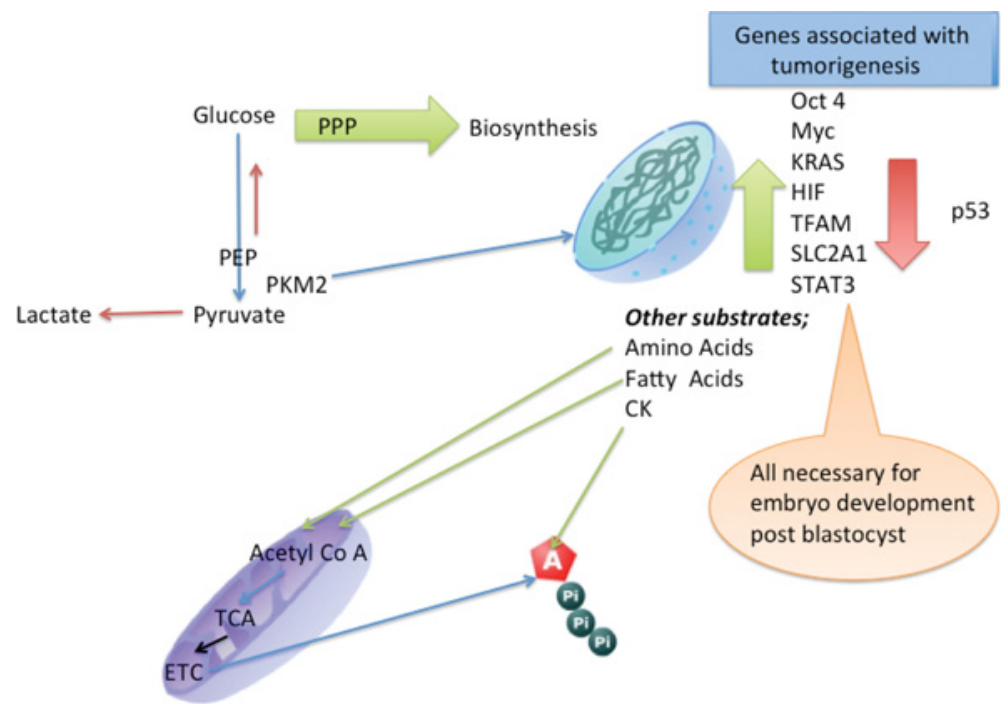

growth; this effect could be overcome by the addition of exogenous sources of high fat [36]. This may contribute to the trend for high triacylglycerol storage being associated with higher levels of cancer development and progression [37].

\section{Early embryos}

One of the key features of the oocyte and early embryo of many mammalian species is the presence of a significant store of endogenous triacylglycerol (reviewed by $[38,39])$. There is now growing evidence for the importance of triacylglycerol metabolism for early embryos in the cow [40], pig [5] and mouse [41], all of which have shown that pharmacological inhibition of $\beta$-oxidation at various stages of preimplantation development significantly reduces ongoing embryo viability. The recent interest in fatty acid metabolism in early embryos has been driven by the soaring global rates of obesity, such that there are now more adults globally who are overweight than underweight. There is growing evidence that obesity has a negative impact on female reproductive health [42]. A number of reports have identified that the follicular environment, which supports development of the unfertilized oocyte, of overweight and obese women is enriched for a number of metabolites, notably triacylglycerol [43]. The impact of development in an enriched lipid environment is not yet clear; however, Van Hoeck et al. [44] have recently described in a bovine model that exposure to elevated NEFAs during oocyte development reduced blastocyst development and viability. Crucially, embryos that originate from oocytes matured in a high-NEFA environment do not use glucose at the blastocyst stage, and the embryonic 'Warbug effect' that we have described above is inhibited. This observation raises the question of whether there may be value in targeting the $\beta$ oxidation pathway in the metabolic manipulation of cancer cells. Indeed, in a promising study, Tirado-Velez et al. [45] recently reported that human myeloma cells are sensitive to inhibition of fatty acid metabolism.

\section{Parallels in regulation}

In the sections above, we described some parallels and differences in overall fuel utilization and specific substrate depletion and production between cancer cells and early embryos. However, there is evidence for parallels in the underlying regulatory mechanisms. To give one example, Christofk et al. [46] proposed a role for PK (pyruvate kinase) in cancer cell progression. Normally, PK catalyses the final step of glycolysis, where it dephosphorylates PEP (phosphoenolpyruvate), forming ATP and pyruvate. There is particular interest in the isoform PKM2, which is expressed by all cancer cells studied to date [47]. Vander Heiden et al. [47] proposed that PKM2 leads to an 'alternative' glycolysis where PEP phosphorylates the enzyme PGAM (phosphoglycerate mutase). It is widely believed that this occurs because PKM2 is a less active isoform of PK [46], thus reducing the rate of formation of pyruvate. Phosphorylation of PGAM increases its activity, leading to feedback where PKM2 is bypassed, since removal of phosphoryl from PEP results in the production of pyruvate. Essentially, 
this produces pyruvate from PEP without transferring the phosphoryl group to ADP and so bypasses an ATP production step in glycolysis, while enhancing the pathway overall. It is therefore conceivable that a consequence of such a strategy is increased glucose uptake and phosphorylation, which can then be diverted into the PPP.

This possible role for PKM2 in metabolic regulation in cancer cells is particularly relevant, since PKM2 is the embryonic form of PK. This may partially explain the early embryo's preference for pyruvate as a fuel source during early cleavage. Embryos express PKM2 (fetal exon $\mathrm{D}$, resulting in the M2 variant) similarly to tumour cells and expression increases at the blastocyst stage [48], the time at which the early embryo markedly increases glucose uptake and glycolysis, yet still relies on oxidative processes for the production of $80-90 \%$ of its ATP. Interestingly, serine has been proposed recently to activate PKM2 [49]. This is particularly relevant since early embryos and some cancer cell types [50] consume significant amounts of serine.

In addition to its catalytic role, PKM2 can localize to the nucleus where it acts as a transcription factor and is involved in histone modification and epigenetic regulation [51]. Of particular interest is the observation that PKM2 has been implicated in the transcriptional regulation of Oct4, $M y c$, KRAS, HIF, TFAM, SLC2A1, STAT3 and $p 53$ [52]; all genes that play a well-defined role in the progression of many cancers. However, many of these genes are also required for normal embryonic development (Figure 2).

\section{Conclusion}

On the strength of a significant body of research, there do appear to be a number of metabolic parallels between early embryos and cancer cells. However, it is necessary to be cautious of over-interpretation, particularly with regard to cancer cell metabolism. 'Cancer' is a broad term, and there is wide variation between different types. Furthermore, much of the data on cancer cell metabolism have of necessity been derived from cancer cells in vitro; the situation may well be different in cancer cells in vivo, where there will be complex cell-cell and tissue-tissue interactions as well as an intricate network of signals present in a functioning physiological system. By contrast, the early embryo can be considered an 'autonomous system', with very definite and reproducible end points. As the altered metabolic activity that characterizes many tumours is recognized as an integral part of the tumorigenic pathway, rather than a mere by-product of cell transformation, the early embryo may represent a good model to enhance our understanding of metabolic strategies of 'de-differentiated' cells including cancer cells.

\section{Acknowledgements}

We thank Professor Henry Leese for constructive comments and helpful discussions during the preparation of this paper.

\section{References}

1 Warburg, 0. (1956) On the origin of cancer cells. Science $\mathbf{1 2 3}, 309-314$

2 Gatenby, R.A. and Gillies, R.J. (2004) Why do cancers have high aerobic glycolysis? Nat. Rev. Cancer 4, 891-899

3 Vander Heiden, M.G., Cantley, L.C. and Thompson, C.B. (2009) Understanding the Warburg effect: the metabolic requirements of cell proliferation. Science 324, 1029-1033

4 Fridhandler, L. (1961) Pathways of glucose metabolism in fertilized rabbit ova at various pre-implantation stages. Exp. Cell Res. 22, 303-316

5 Sturmey, R.G. and Leese, H.J. (2003) Energy metabolism in pig oocytes and early embryos. Reproduction 126, 197-204

6 Houghton, F.D., Thompson, J.G., Kennedy, C.J. and Leese, H.J. (1996) oxygen consumption and energy metabolism of the early mouse embryo. Mol. Reprod. Dev. 44, 476-485

7 Thompson, J.G., Partridge, R.J., Houghton, F.D., Cox, C.I. and Leese, H.J. (1996) Oxygen uptake and carbohydrate metabolism by in vitro derived bovine embryos. J. Reprod. Fertil. 106, 299-306

8 Leese, H.J. (2012) Metabolism of the preimplantation embryo: 40 years on. Reproduction 143, 417-427

9 Seli, E., Vergouw, C.G., Morita, H., Botros, L., Roos, P., Lambalk, C.B., Yamashita, N., Kato, O. and Sakkas, D. (2010) Noninvasive metabolomic profiling as an adjunct to morphology for noninvasive embryo assessment in women undergoing single embryo transfer. Fertil. Steril. 94, 535-542

10 Houghton, F.D., Hawkhead, J.A., Humpherson, P.G., Hogg, J.E., Balen, A.H., Rutherford, A.J. and Leese, H.J. (2002) Non-invasive amino acid turnover predicts human embryo developmental capacity. Hum. Reprod. 17, 999-1005

11 Chen, Z., Lu, W., Garcia-Prieto, C. and Huang, P. (2007) The Warburg effect and its cancer therapeutic implications. J. Bioenerg. Biomembr. 39, 267-274

12 Martin, K.L. and Leese, H.J. (1995) Role of glucose in mouse preimplantation embryo development. Mol. Reprod. Dev. 40, 436-443

13 Martin, K.L. and Leese, H.J. (1999) Role of developmental factors in the switch from pyruvate to glucose as the major exogenous energy substrate in the preimplantation mouse embryo. Reprod. Fertil. Dev. 11, 425-433

14 Swain, J.E., Bormann, C.L., Clark, S.G., Walters, E.M., Wheeler, M.B. and Krisher, R.L. (2002) Use of energy substrates by various stage preimplantation pig embryos produced in vivo and in vitro. Reproduction 123, 253-260

15 Gardner, D.K., Wale, P.L., Collins, R. and Lane, M. (2011) Glucose consumption of single post-compaction human embryos is predictive of embryo sex and live birth outcome. Hum. Reprod. 26, 1981-1986

16 Gardner, D.K. and Leese, H.J. (1990) Concentrations of nutrients in mouse oviduct fluid and their effects on embryo development and metabolism in vitro. J. Reprod. Fertil. 88, 361-368

17 Krisher, R.L. and Prather, R.S. (2012) A role for the Warburg effect in preimplantation embryo development: metabolic modification to support rapid cell proliferation. Mol. Reprod. Dev. 79, 311-320

18 Lu, S.C. (1999) Regulation of hepatic glutathione synthesis: current concepts and controversies. FASEB J. 13, 1169-1183

19 Guerin, P., El Mouatassim, S. and Menezo, Y. (2001) Oxidative stress and protection against reactive oxygen species in the pre-implantation embryo and its surroundings. Hum. Reprod. Update 7, 175-189

20 Javed, M.H. and Wright, Jr, R.W. (1991) Determination of pentose phosphate and Embden-Meyerhof pathway activities in bovine embryos. Theriogenology 35, 1029-1037

21 DeBerardinis, R.J., Mancuso, A., Daikhin, E., Nissim, I., Yudkoff, M., Wehrli, S. and Thompson, C.B. (2007) Beyond aerobic glycolysis: transformed cells can engage in glutamine metabolism that exceeds the requirement for protein and nucleotide synthesis. Proc. Natl. Acad. Sci. U.S.A. 104 19345-19350

22 Newsholme, E.A., Crabtree, B. and Ardawi, M.S. (1985) The role of high rates of glycolysis and glutamine utilization in rapidly dividing cells. Biosci. Rep. 5, 393-400

23 Teicher, B.A., Linehan, W.M. and Helman, L.J. (2012) Targeting cancer metabolism. Clin. Cancer Res. 18, 5537-5545

24 Sauer, L.A., Dauchy, R.T., Nagel, W.O. and Morris, H.P. (1980) Mitochondrial malic enzymes. Mitochondrial NAD(P) + -dependent malic enzyme activity and malate-dependent pyruvate formation are progression-linked in Morris hepatomas. J. Biol. Chem. 255, 3844-3848

25 Rajagopalan, K.N. and DeBerardinis, R.J. (2011) Role of glutamine in cancer: therapeutic and imaging implications. J. Nucl. Med. $\mathbf{5 2}$ 1005-1008 
26 Carney, E.W. and Bavister, B.D. (1987) Stimulatory and inhibitory effects of amino acids on the development of hamster eight-cell embryos in vitro. J. In Vitro Fertil. Embryo Transfer 4, 162-167

27 Chatot, C.L., Ziomek, C.A., Bavister, B.D., Lewis, J.L. and Torres, I. (1989) An improved culture medium supports development of random-bred 1-cell mouse embryos in vitro. J. Reprod. Fertil. 86, 679-688

28 Petters, R.M., Johnson, B.H., Reed, M.L. and Archibong, A.E. (1990) Glucose, glutamine and inorganic phosphate in early development of the pig embryo in vitro. J. Reprod. Fertil. 89, 269-275

29 Devreker, F., Winston, R.M. and Hardy, K. (1998) Glutamine improves human preimplantation development in vitro. Fertil. Steril. 69, 293-299

30 Humpherson, P.G., Leese, H.J. and Sturmey, R.G. (2005) Amino acid metabolism of the porcine blastocyst. Theriogenology 64, 1852-1866

31 Sturmey, R.G., Hawkhead, J.A., Barker, E.A. and Leese, H.J. (2009) DNA damage and metabolic activity in the preimplantation embryo. Hum. Reprod. 24, 81-91

32 Rieger, D., Loskutoff, N.M. and Betteridge, K.J. (1992) Developmentally related changes in the metabolism of glucose and glutamine by cattle embryos produced and co-cultured in vitro. J. Reprod. Fertil. 95, 585-595

33 Leese, H.J., Conaghan, J., Martin, K.L. and Hardy, K. (1993) Early human embryo metabolism. BioEssays 15, 259-264

34 Jain, M., Nilsson, R., Sharma, S., Madhusudhan, N., Kitami, T., Souza, A.L., Kafri, R., Kirschner, M.W., Clish, C.B. and Mootha, V.K. (2012) Metabolite profiling identifies a key role for glycine in rapid cancer cell proliferation. Science 336, 1040-1044

35 Biswas, S., Lunec, J. and Bartlett, K. (2012) Non-glucose metabolism in cancer cells-is it all in the fat? Cancer Metastasis Rev. 31, 689-698

36 Nomura, D.K., Long, J.Z., Niessen, S., Hoover, H.S., Ng, S.W. and Cravatt, B.F. (2010) Monoacylglycerol lipase regulates a fatty acid network that promotes cancer pathogenesis. Cell 140, 49-61

37 Calle, E.E. and Kaaks, R. (2004) Overweight, obesity and cancer: epidemiological evidence and proposed mechanisms. Nat. Rev. Cancer 4, 579-591

38 Sturmey, R.G., Reis, A., Leese, H.J. and McEvoy, T.G. (2009) Role of fatty acids in energy provision during oocyte maturation and early embryo development. Reprod. Domest. Anim. 44 (Suppl. 3), 50-58

39 McKeegan, P.J. and Sturmey, R.G. (2011) The role of fatty acids in oocyte and early embryo development. Reprod. Fertil. Dev. 24, 59-67

40 Ferguson, E.M. and Leese, H.J. (2006) A potential role for triglyceride as an energy source during bovine oocyte maturation and early embryo development. Mol. Reprod. Dev. 73, 1195-1201

41 Dunning, K.R., Cashman, K., Russell, D.L., Thompson, J.G., Norman, R.J. and Robker, R.L. (2010) $\beta$-Oxidation is essential for mouse oocyte developmental competence and early embryo development. Biol. Reprod. 83, 909-918
42 Lash, M.M. and Armstrong, A. (2009) Impact of obesity on women's health. Fertil. Steril. 91, 1712-1716

43 Robker, R.L., Akison, L.K., Bennett, B.D., Thrupp, P.N., Chura, L.R., Russell, D.L., Lane, M. and Norman, R.J. (2009) Obese women exhibit differences in ovarian metabolites, hormones, and gene expression compared with moderate-weight women. J. Clin. Endocrinol. Metab. 94, 1533-1540

44 Van Hoeck, V., Leroy, J.L., Arias-Alvarez, M., Rizos, D., Gutierrez-Adan, A., Schnorbusch, K., Bols, P.E., Leese, H.J. and Sturmey, R.G. (2013) Oocyte developmental failure in response to elevated non-esterified fatty acid concentrations: mechanistic insights. Reproduction 145, 33-44

45 Tirado-Vélez, J.M., Joumady, I., Sáez-Benito, A., Cózar-Castellano, I. and Perdomo, G. (2012) Inhibition of fatty acid metabolism reduces human myeloma cells proliferation. PLoS ONE 7, e46484

46 Christofk, H.R., Vander Heiden, M.G., Harris, M.H., Ramanathan, A., Gerszten, R.E., Wei, R., Fleming, M.D., Schreiber, S.L. and Cantley, L.C. (2008) The M2 splice isoform of pyruvate kinase is important for cancer metabolism and tumour growth. Nature 452, 230-233

47 Vander Heiden, M.G., Locasale, J.W., Swanson, K.D., Sharfi, H., Heffron, G.J., Amador-Noguez, D., Christofk, H.R., Wagner, G., Rabinowitz, J.D., Asara, J.M. and Cantley, L.C. (2010) Evidence for an alternative glycolytic pathway in rapidly proliferating cells. Science 329, 1492-1499

48 Redel, B.K., Brown, A.N., Spate, L.D., Whitworth, K.M., Green, J.A. and Prather, R.S. (2012) Glycolysis in preimplantation development is partially controlled by the Warburg effect. Mol. Reprod. Dev. 79, 262-271

49 Chaneton, B., Hillmann, P., Zheng, L., Martin, A.C., Maddocks, O.D., Chokkathukalam, A., Coyle, J.E., Jankevics, A., Holding, F.P., Vousden, K.H. et al. (2012) Serine is a natural ligand and allosteric activator of pyruvate kinase M2. Nature 491, 458-462

50 Possemato, R., Marks, K.M., Shaul, Y.D., Pacold, M.E., Kim, D., Birsoy, K., Sethumadhavan, S., Woo, H.K., Jang, H.G., Jha, A.K. et al. (2011) Functional genomics reveal that the serine synthesis pathway is essential in breast cancer. Nature 476, 346-350

51 Yang, W., Xia, Y., Hawke, D., Li, X., Liang, J., Xing, D., Aldape, K., Hunter, T., Alfred Yung, W.K. and Lu, Z. (2012) PKM2 phosphorylates histone $\mathrm{H} 3$ and promotes gene transcription and tumorigenesis. Cell 150, 685-696

52 Luo, W. and Semenza, G.L. (2012) Emerging roles of PKM2 in cell metabolism and cancer progression. Trends Endocrinol. Metab. 23, 560-566

Received 14 December 2012

doi:10.1042/BST20120352 